\title{
Reabilitação do guia canino complementar ao tratamento harmônico e estético com prótese parcial fixa livre de metal
}

\author{
Rehabilitation of the canine guide complementary to the harmonic \\ and aesthetic treatment with fixed partial denture of metal
}

\begin{abstract}
RESUMO
Introdução: A reabilitação estética de dentes anteriores com próteses cerâmicas livres de metal proporciona excelência estética principalmente pela ausência da margem metálica. Pacientes que apresentam oclusão adequada e ausência de hábitos parafuncionais possuem menor chances de falhas catastróficas em movimentos excêntricos e bordejantes durante as excursões mandibulares.

Objetivo: evidenciar, por meio de relato de um caso clínico, o reestabelecimento da estética e função de dentes anteriores, utilizando restaurações cerâmicas livres de metal. Conclusões: A utilização de cerâmicas livres de metal permitiu uma estética natural e funcional com ótimas propriedades ópticas, integrando as restaurações ao sorriso do paciente.

Palavras-chave: Reabilitação oral, metal free, estética dentária.
\end{abstract}

\section{ABSTRACT}

Introduction: The aesthetic rehabilitation of anterior teeth with metal-free ceramic prosthesis provides aesthetic excellence mainly due to the absence of metal margin. Patients with adequate occlusion and absence of parafunctional habits have a lower chance of catastrophic failure in eccentric movements and contours during mandibular excursions.

Aim: to report, through a clinical case report, reestablishment of aesthetics and function of anterior teeth, using metal free ceramic restorations.

Conclusions: The use of free metal ceramics allowed a natural and functional aesthetic with excellent optical properties, integrating the restorations to the patient's smile. Key words: Oral rehabilitation, metal free, dental aesthetics.

\section{INTRODUÇÃO}

A odontologia está cada vez mais ligada à estética como na atualidade. Dentes brancos, alinhados, sorriso agradável. Tudo isso tem sido objeto de desejo. É por essa razão que a estética tem sido cada vez mais importante. Existem procedimentos específicos para cada caso, o cirurgião-dentista deve ser minucioso com a elaboração de um plano de tratamento com uma integração entre, paciente, técnico em prótese, cirurgião dentista, tornando-se imprescindível para obter o sucesso no tratamento, reabilitador com diagnostico, indicação e planejamento, e conhecimento da técnica operatória, das características de cada sistema indireto, cor, anatomia dentaria e conhecimento de suas propriedades físico-mecânicas dentre outros fatores ${ }^{1,2}$.

\section{Vinicius Coronado Barros* Igor Coronado Barros ${ }^{* *}$ \\ Joaquim mesquita Neto ${ }^{* * * *}$}

* Cursando especialização em prótese dentaria FFO-USP, email: vini_sc10@hotmail.com ••Cursando especialização em cirurgia e traumatologia bucomaxilofaciais pela FFO-USP, email: igorcoronado92@outlook.com .. Especialista em prótese dentaria pelo instituto metodista.

Autor e endereço para contato:

Vinicius Coronado Barros

Rua Rogerio Giorgi, 830 -Santo André - SP

CEP: 09112130

Email:

Vini_sc10@hotmail.com

Telefone: 9 6465-2501/4978-2092

Submetido em: 17-8-2017

Aceito em: 11-12-2018 
Contudo os sistemas cerâmicos surgiram e evoluíram para desempenhar um papel importante frente à desvantagem de um substrato metálico ${ }^{3,4}$. Isto é consequência do aprimoramento das propriedades físicas das cerâmicas odontológicas, melhoria e estabelecimento das técnicas adesivas e o aperfeiçoamento dos cirurgiões dentistas ${ }^{2,3}$.

Então, as restaurações livres de metal, ditas metal free. São restaurações que não apresentam infraestrutura metálica, permitindo a passagem de luz pela restauração e boa resistência à fratura ${ }^{3,5,9}$. A porcelana odontológica convencional é uma cerâmica vítrea, que possui, como principais componentes químicos, minerais cristalinos tais como o feldspato, quartzo, alumina e, eventualmente, caolin e uma matriz vítrea, sendo considerada como material restaurador que possui características estéticas comparáveis as dos dentes naturais ${ }^{4}$. Outro fator de grande importância é a conformação da guia anterior que seja benéfica ao sistema mastigatório, evitando contatos posteriores durante os movimentos mandibulares excêntricos ${ }^{5,16}$. A ausência de uma oclusão funcional ideal pode destruir a estrutura dentária necessária para a estabilidade oclusal, proteção mútua, função, fonética e estética da guia anterior e desordens temporomandibulares ${ }^{6,16}$.

O conceito do guia do dente canino preconiza o dente canino guiar a desoclusão do lado de trabalho e balanceio nos movimentos laterais ${ }^{20}$. Outro tipo de padrão de desoclusão pode ocorrer na população, á função em grupo ${ }^{20}$. A guia incisal define-se como a desoclusão dos dentes posteriores e contato dos dentes anteriores no movimento de protrusão mandibular. Este movimento é guiado pela face palatina dos incisivos superiores e pela borda incisal dos incisivos inferiores ${ }^{20}$. Este tipo de oclusão é definido pela proteção dos dentes posteriores pelos dentes anteriores nos movimentos excursivos da mandíbula, e proteção dos dentes anteriores pelos dentes posteriores em máxima intercuspidação, sem interferência ${ }^{16,20}$.

Será apresentado e discutido nesse caso clínico no qual foi necessária a reabilitação protética dos elementos 13,12,11,21,22 e 23. A partir da confecção de uma prótese fixa livre de metal, e reconstrução do guia anterior, com o objetivo de proporcionar ao paciente a boa função, estética, fonética e o perfeito equilíbrio fisiológico da oclusão.

Os materiais mais resistentes falham pela falta de translucidez. Os mais estéticos, por sua vez, sofrem maior risco de fratura ${ }^{7,8}$. Por isso, próteses metalocerâmicas e as livres de metal continuam dividindo as preferências e as indicações dos cirurgiões dentistas. As cerâmicas, com as melhores propriedades mecânicas, In-Ceram alumínio e In-Ceram zircônia ${ }^{10,11}$. Estes sistemas tem alta resistência e oferecem uma ampla possibilidade de indicações e aplicação clínica, por exemplo, em próteses unitárias, infraestrutura para pontes fixas e coroas sobre implantes, além de não apresentarem correntes galvânicas, o que contribui para a manutenção da saúde periodontal e pulpar, diminuição de acúmulo de placa, excelência estética, pela translucidez, opacidade, opalescência e fluorescência, além de serem superiores em relação às metalocerâmicas nos quesitos: corrosão, e biocompatibilidade ${ }^{11,12,13}$. Então para o paciente ser considerado apto a receber restaurações protéticas livres de metal, deve possuir oclusão adequada e ausência de hábitos parafuncionais, para que estas não se fraturem em movimentos excêntricos e bordejantes durante as excursões mandibulares ${ }^{5}$.

Diante das evidências clínicas e científicas relatadas acima, objetivou-se neste estudo descrever um relato de caso clínico, onde a paciente foi submetida a reabilitação protética dos incisivos e caninos superiores, preconizando o uso materiais cerâmicos livres de metal. 


\section{RELATO DE CASO}

Paciente, leucoderma, gênero feminino S.M.B. 35 anos de idade, compareceu a clínica particular queixando-se da aparência de seu sorriso. Ao exame clinico e radiográfico inicial foi observado: Uso de aparelho ortodôntico, dentes com diastemas presentes, hiperplasia gengival, sem perda de inserção, perda de estrutura dental em algumas faces. Não relatava qualquer sintomatologia articular (FIGURA 1).

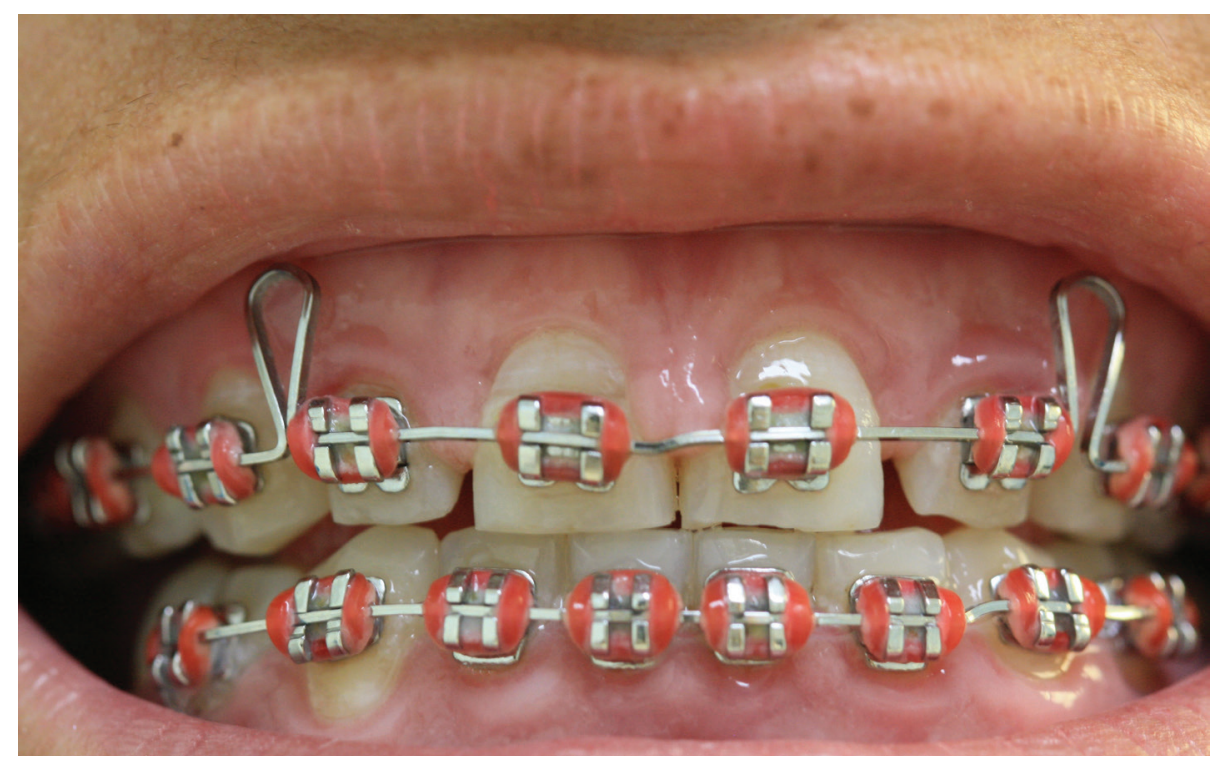

FIGURA 1

Optou-se pela confecção de coroas metal free nos elementos 11,12,13,21,22 e 23. Pelo fato de sua durabilidade a longos períodos, estabilidade de cor, resistência ao desgaste e abrasão.

Então inicialmente, foi feito um enceramento de diagnostico. Foi sugerida a utilização do sistema In-Ceram Zirconia. Posteriormente foram realizados os preparos dentais, do tipo coroa total, com termino cervical em chanfro (ponta diamantada 4138, KG Sorensen, Brasil). (FIGURA 2).

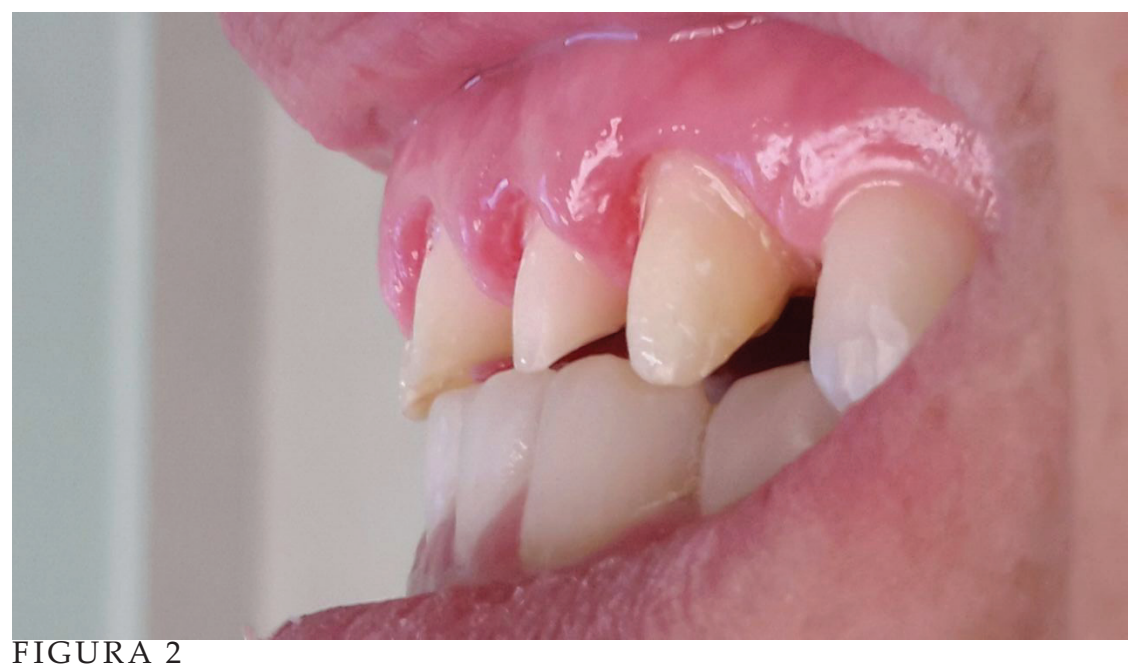


Os provisórios em resina acrílica foram confeccionados em laboratório e foram reembasados (Resina Acrílica Dencor) e cimentados provisoriamente com cimento de óxido de zinco sem eugenol (Temp Bond NE - Kerr, EUA).

Na terceira consulta clínica, realizou-se o afastamento gengival com duplo fio (U1trapack 000 e 00 - Ultradent, EUA) e moldou-se com silicone de adição (Silagum - DMG, Alemanha) pela técnica de impressão dupla. Foi vazado o modelo em gesso tipo IV (Durone - Dentsply), troquelizado e enviado ao laboratório de prótese para a confecção dos coppings em zircônia para as coroas totais.

Provaram-se os coppings e avaliou-se internamente, externamente e sua espessura, adaptação marginal e cor do copping que pode trazer benefícios, no sentido de obter um melhor resultado estético final. (FIGURA 3,4 e 5) E realizou-se uma moldagem de transferência com silicone de adição (Silagum - DMG, Alemanha) e selecionou-se a cor A1 com auxilio de uma escala (Escala Vita Classica, Vita-Germany).

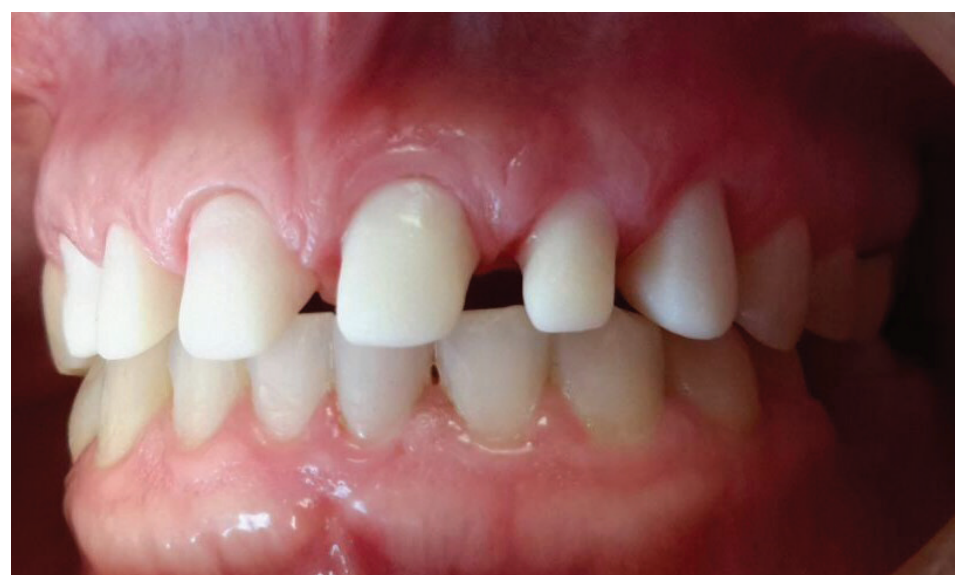

FIGURA 3

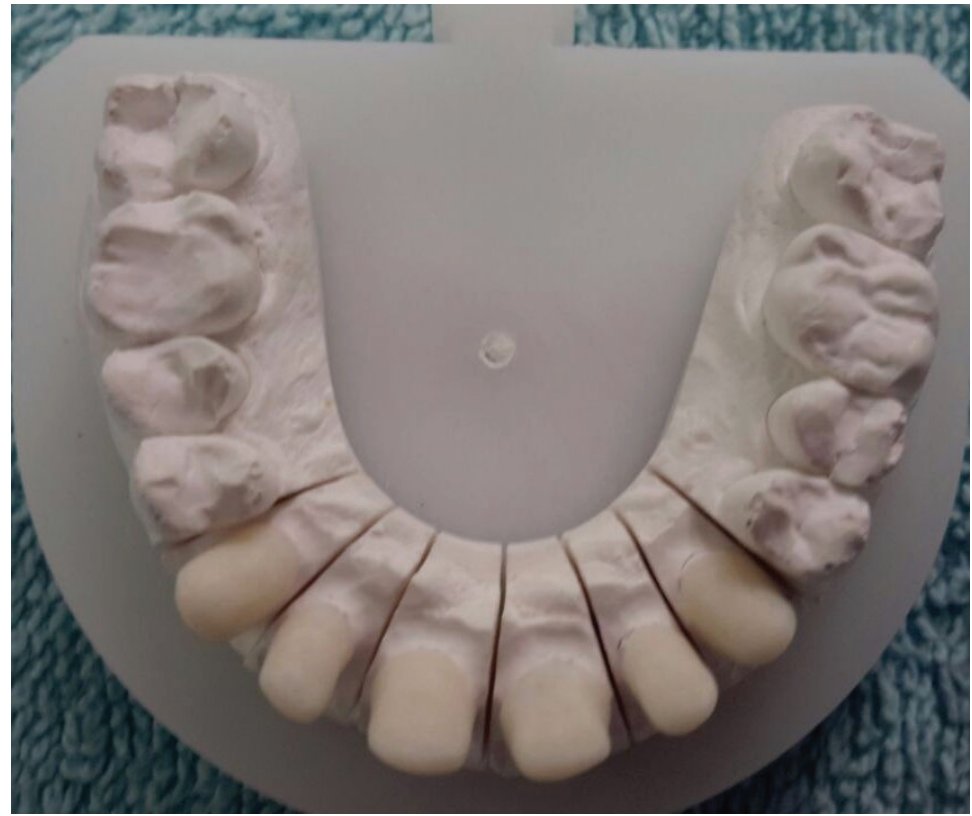

FIGURA 4 


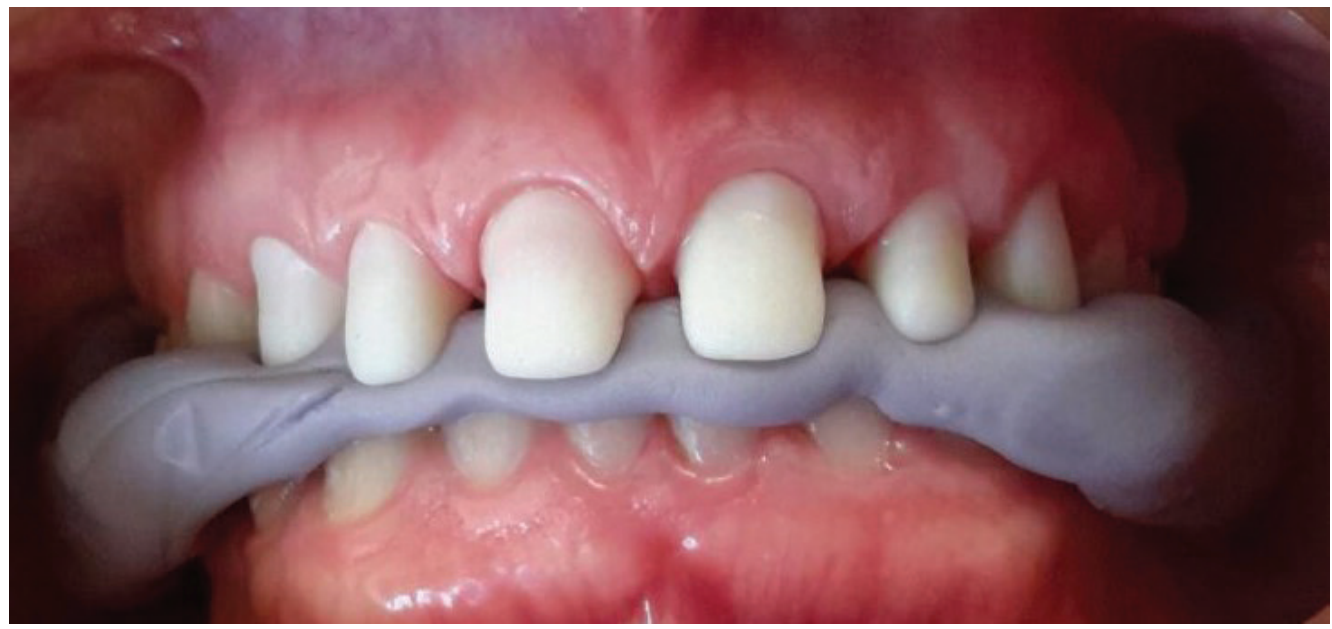

FIGURA 5

Após a confecção das coroas protéticas, removeram-se os provisórios, realizou-se uma profilaxia dos preparos dentais e prova das coroas avaliando-se a adaptação marginal e ponto de contato.

Realizou-se um isolamento relativo do campo operatório com a intenção de impedir que os fluidos gengivais prejudiquem a adesão. Foi utilizada cimentação convencional com cimento de fosfato de zinco (Cimento Fosfato de Zinco SS White) manipulado segundo a recomendação do fabricante.

Avaliaram-se os cotantos oclusais com carbono Acuufilm II (PARKELL-EUA) em máxima intercuspidação habitual e nas guias dos caninos e guia incisal anterior e canino realizando os ajustes oclusais necessários a ponta diamantada 2135 FF (KG Soresen, Brasil) acabamento e polimento final com sistema de borrachas para cerâmica (Labordental, EUA).

O resultado final demonstrou uma estética natural e funcional com ótimas propriedades ópticas. Obteve-se ausência de interferência do lado de trabalho, tendo como guia o dente canino e no lado de balanceio a desoclusão. Desoclusão dos dentes posteriores nos movimentos protrusivos. (FIGURA 6 e 7). Alem da completa integração das restaurações com sorriso do paciente. (FIGURA 8 e 9).

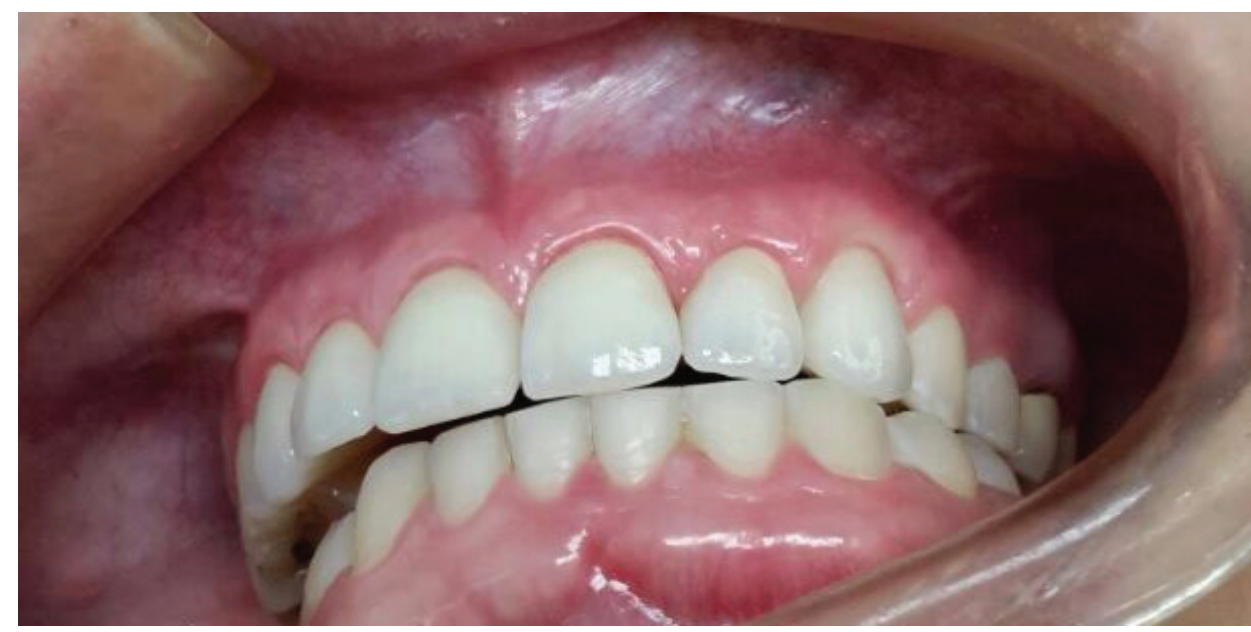

FIGURA 6 


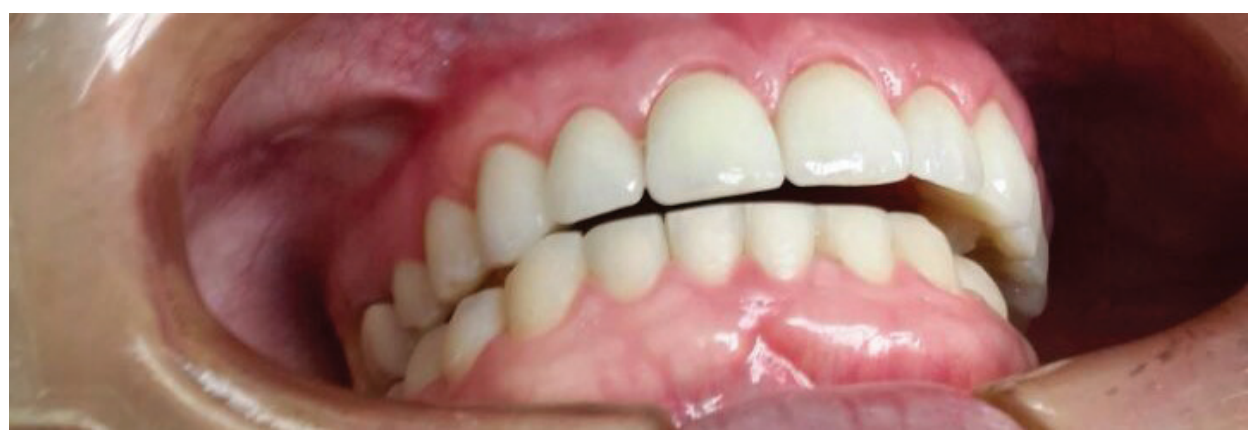

FIGURA 7

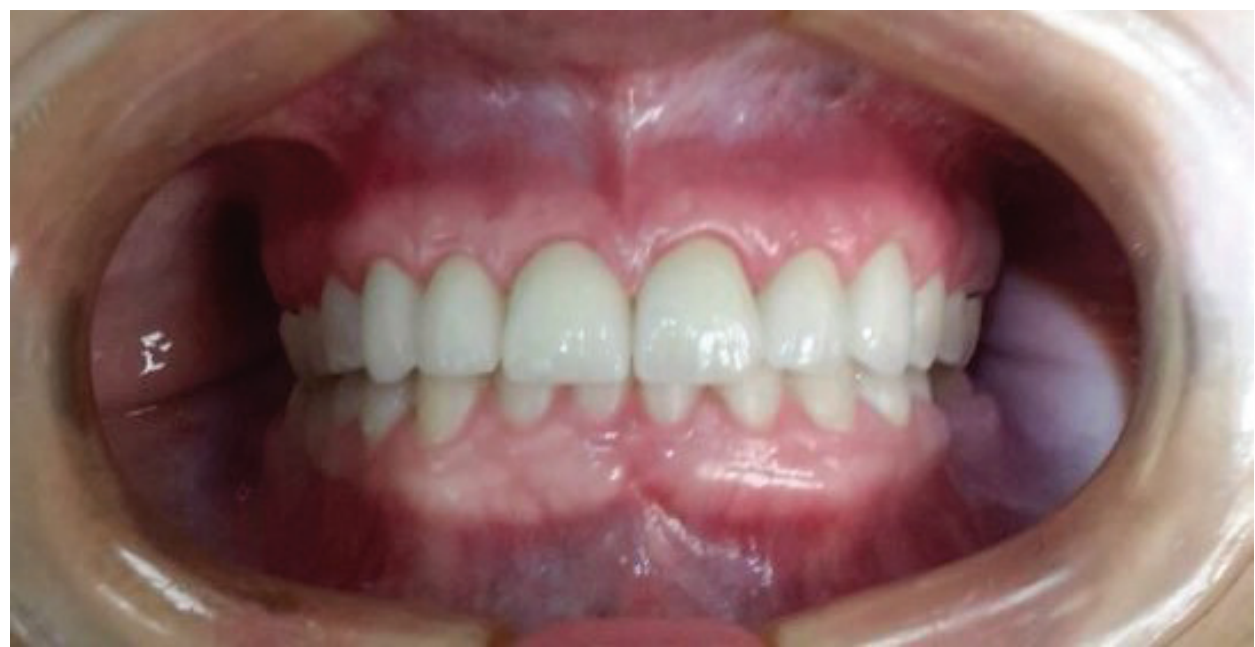

FIGURA 8

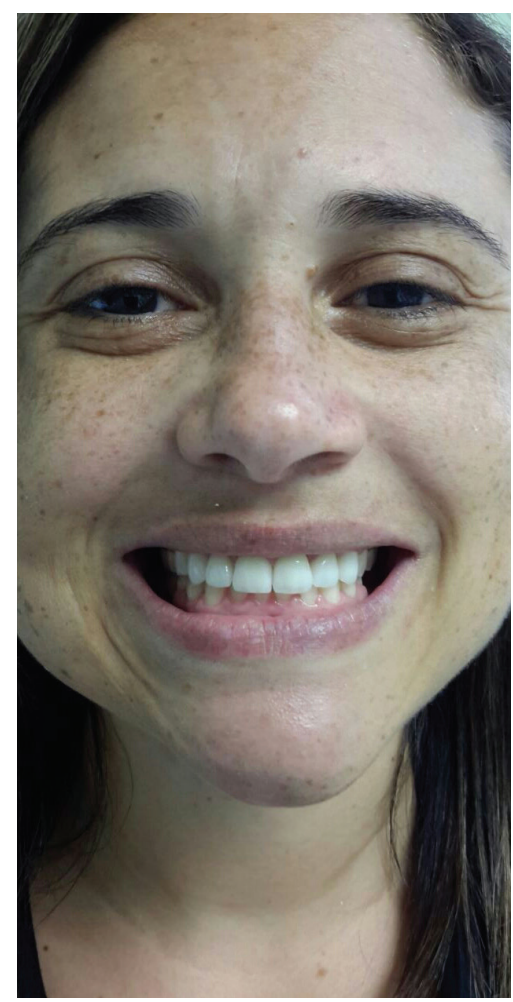

FIGURA 9 
Foi feita a proservação deste paciente após um ano da reabilitação, e as imagens foram realizadas e documentadas com câmera intra-oral Gruposky. E comprovou-se o sucesso do procedimento clinico reabilitador utilizando o sistema In-Ceram Zirconia.

\section{DISCUSS ÃO}

Os materiais mais resistentes limitam o resultado estético pela ausência de translucidez do substrato metálico ${ }^{10}$. As resinas de macropartículas apresentavam lisura superficial insatisfatória. As resinas microparticuladas têm como inconveniente um alto índice de contração de polimerização devido a pouca porcentagem de carga em peso dessas resinas ${ }^{20,21}$. Nenhuma resina composta atingiu excelência de um material restaurador ideal ${ }^{21}$. Uma falha que acontece constantemente nas restaurações de resinas compostas é a ruptura da interface adesiva, que geralmente ocorre devido a uma das principais características negativas das resinas: a contração de polimerização ${ }^{21}$. Com isso a confecção de coroas com sistemas cerâmicas metal free mostra-se como excelente alternativa restauradora, com potencialidade estética superior. Isso se deve pela possibilidade da realização de estratificação de cores de modo mais eficiente, permitindo um aspecto mais natural e harmonioso com os dentes adjacentes ${ }^{2}$. Porém os mais estéticos, por sua vez, sofre maior risco de fratura ${ }^{11}$. Por isso, próteses metalocerâmicas e as livres de metal continuam dividindo as preferências e indicações dos cirurgiões dentistas.

Assim, deste modo, a avaliação das expectativas do paciente e o conhecimento das possíveis soluções terapêuticas na área odontológica, são fundamentais antes de iniciar qualquer planejamento reabilitador ${ }^{1 .}$.

As próteses parciais fixas metal free, são relativamente novas e estão em frequente crescimento na odontologia, mas pode-se algumas contraindicações, tais como, bruxismo, pacientes com higiene oral deficiente, inflamação gengival ${ }^{2,4-6}$. Além de que a capacidade de suportar cargas é fortemente limitada, sendo fator crítico para pontes de ampla extensão e pontes posteriores ${ }^{4}$. Além da quantidade de desgaste correta, o profissional capacitado deve atentar a precisão da técnica e do preparo dental que deve apresentar condições mecânicas de estabilidade e retenção para manter a coroa assentada e, consequentemente, ter sucesso no tratamento clínico ${ }^{7}$. A utilização de sistemas cerâmicos tem-se mostrado como alternativa de excelente resultado estético ${ }^{4}$. As cerâmicas disponíveis atualmente no mercado apresentam vantagens significativas sobre as próteses metalocerâmicas e resinas compostas $^{21}$. Próteses livres de metal não possuem zona de sombreamento na região cervical, além de não apresentarem correntes galvânicas, o que contribui para a manutenção da saúde periodontal e pulpar. Incluindo a diminuição de acúmulo de placa, excelência estética, em especial pela translucidez, opacidade, opalescência e fluorescência, além de permitir a reprodução dos dentes e estruturas anatômicas com textura e forma individualizada, além de serem superiores em relação às metalocerâmicas nos quesitos: corrosão, e biocompatibilidade ${ }^{12,13}$. Com isso, para o sucesso do trabalho reabilitador protético, a oclusão do paciente deve ser equilibrada sem nenhum aspecto parafuncional ${ }^{3}$. Então para o paciente ser considerado apto a receber restaurações protéticas livres de metal, deve possuir oclusão adequada e ausência de hábitos parafuncionais, para que estas não se fraturem em movimentos excêntricos e bordejantes durante os movimentos mandibulares ${ }^{14}$. 
Ao se estabelecer a guia anterior, tem-se como finalidade obter o equilíbrio do sistema neuromuscular e da articulação temporomandibular (ATM) e buscar o tratamento mais duradouro e estável ${ }^{15,17}$. Uma vez reconhecida a necessidade de desoclusão anterior, deve ser estabelecido o contorno, posição, inclinação e trespasse adequados dos dentes anteriores, para alcançar os objetivos ${ }^{6}$. Logo, para que o paciente apresente indicação para esse tipo de procedimento protético, é necessária uma oclusão equilibrada, sem patologias oclusais ${ }^{3}$.

As cerâmicas que possui uma das melhores propriedades mecânicas como In-Ceram Alumínio e In-Ceram Zircônia ${ }^{14}$. Estes sistemas tem alta resistência e oferecem uma ampla possibilidade de aplicações clinicas ${ }^{1}$. Independentemente da técnica de cimentação empregada, quando se utilizou cerâmicas à base de zircônia, a prótese parcial fixa apresentaram as mais altas taxas de longevidade, segundo a literatura ${ }^{10,14}$.

A relação entre cirurgião dentista, técnico em prótese dental e paciente na elaboração do planejamento reabilitador foi essencial para o resultado final obtido, nesse caso, o que deve ser um fundamento frequente na clinica ${ }^{1}$.

Neste caso optou-se pela reabilitação estética e funcional com restaurações em cerâmica, principalmente pela durabilidade da cerâmica, comparada a restaurações direta de resina composta. Assim, os cirurgiões-dentistas devem conhecer as diversas formas e sequências de tratamento, que dependem muito do senso clínico do profissional e de sua habilidade em detectar os fatores etiológicos envolvidos. $\mathrm{O}$ uso adequado dos sistemas restauradores indiretos, trás a longevidade, satisfação do paciente, e princípios fundamentais da oclusão aplicados em harmonia ${ }^{6,18,19 .}$

\section{CONCLUSÃO}

A prótese parcial fixa, metal free é uma alternativa eficaz e segura para fins estéticos e terapêuticos na odontologia, devendo ser realizada por profissionais capacitados. É necessário o planejamento adequado e análise criteriosa sobre aspectos estéticos e harmônicos da face do paciente, como também de sua oclusão.

\section{Agradecimentos}

Os autores gostariam de agradecer os técnicos em prótese dental, pela realização desse relato de caso clinico apresentado.

\section{REFERÊNCIAS}

1. Taciana Barcellos Carvalheira, Frederico dos reis Goyata, Carlos Roberto Teixeira Rodrigues, Maria Cristina Almeida Souza. Resolução em estética em dentes anteriores com coroas totais livres de metais, relato de caso clinico. Int j.dent, v.2,n.11,p.102-106, 2010.

2. Moura, R. B. B.; Santos, T. C. R. Sistemas cerâmicos metal free: tecnologia CAD/CAM - revisão de literatura. Interd, v.1, n.8,p.220-226, 2008.

3. Vandré Taumaturgo de Mesquita, Marianna Hypólito Pompeu, Alexandre Henrique de Moura Dias. O novo conceito em prótese fixa. V.1, n.7,p.26-29, 2013.

4. Ígor Chaves Guimarães Peixoto, Emílio Akaki. Avaliação de próteses parciais fixas em cerâmica pura: uma revisão de literatura. Arq. Brás. Odontologia. v,2.n.4,p.96-103, 2008.

5. Juan Carlos Pontons-Melo, Adilson Yoshio Furuse,César Antunes de Freitas, José Mondelli R Dental Press Estét, Maringá. Reabilitação estética e funcional da guia anterior: uma seqüência lógica e conservadora. $\mathrm{R}$ 
Dental Press Estét, v.4,n.4,p.39-49,2007.

6. Luciana Barbosa Morandi Saint'Clair Batista Rabelo Neto. Reabilitação Oral: Prótese fixa metaloceramica anterior inferior com reconstrução de guia. Relato de caso clinico. Arq bras odontol, v.3,n.1,p.38-43, 2007.

7. Renally Bezerra Wanderley e lima, Ricardo jorge alves figueredo, Ana karina maciel de Andrade, Rosângela Marques Duarte. Otimizando a Estética do Sorriso através de Coroa Cerâmica “Metal Free" - Relato de caso, v.2,n.27,p.165-170,2013.

8. Paulo Frederico Rezentti, Matheus Bortoluzzi Mantovani, Giovani de oliveira correa, Silvia masae de Araujo michida, Cleverson oliveira e silva, Fabiano Carlos marson. Reabilitação estética anterior com coroas metal free: relato de caso clínico. Brazilian Journal of Surgery and Clinical Research-BJSCR. V.3,n.4,p.16-20,2013.

9. Francisca Mariane de Souza Costa; Pedro Henrique Acioly Guedes Peixoto Vieira; Érika Matias Pinto Dinelly; Maurício Pompeu Cariello. Sistemas cerâmicos atuais em zircônia, revisão de literatura. Encontro de Extensão, Docência e Iniciação Científica (EEDIC), 12. 2016, Quixadá. Anais. Quixadá: Centro Universitário Católica de Quixadá, 2016. ISSN: 2446-6042.

10. Renan Mendes de Assis Rolim Hugo Ramalho Sarmento Antonio Carlos Lopes Branco Fernanda Campos Sarina Maciel Braga Pereira Rodrigo Othavio de Assunção e Souza. Desempenho clinico das restaurações livres de metal, relato de caso. V.2,n.17,p.309-318,2013.

11. Rubén Agustín-Panadero, Juan-Luis Román-Rodríguez, Alberto Ferreiroa, María-Fernanda Solá-Ruíz, Antonio Fons-Font. Journal section: Oral Medicine and Pathology Publication Types: Review. Zirconia in fixed rosthesis. A literature review J Clin Exp Dent. V.1,n.6,p.66-73,2014.

12. Alesandra Pereira de Andrade, Angela Mayumi Shimaoka, Roberta Lacerda Falanque, Marcio Garcia dos Santos, Flavio Augusto Merichello dos Santos, Rubens Corte Real de Carvalho. Reabilitação estética com a utilização de um sistema cerâmico do tipo Y-TZP: relato de caso clínico RPG Rev Pós Grad v.1,n.19,p.28-31,2012.

13. Marcio Jose Froxino Bindo, Rogério Goulart da Costa, Eduardo Christiano Caregnatto de Morais, Moira Pedroso Leão, Sávio Marcelo Leite Moreira da Silva. Adaptação marginal em prótese livre de metal, observado por meio da microscopia eletrônica de varredura aos três anos em função. RSBO. V.6,n.2,p.130-134,2009.

14. Nishioka RS, Vasconcelos LGO de, Tanaka KT, Duarte Filho G, Jóias RM. In-ceram Zirconia: prótese parcial fixa. Revista Ibero-americana de Prótese Clínica \& Laboratorial 2004; 6(34):540-4.

15. Okeson JP. Tratamento das desordens temporomandibulares e oclusão. $7^{\mathrm{a}}$ ed. São Paulo: Artes Médicas.

16. Carlos Henrique Ribeiro Rodrigues, Heron de Abreu Rebelo de Matos, José Boaventura Zumaêta Costa. Sitientibus, Disfunção crânio-mandibular: guia canino ou função em grupo?., n.30, p.173-186, jan/jun. 2004

17. E. A. Gomes, W. G. Assunção, E. P. Rocha, P. H. Santos R. José Bonifácio. Cerâmicas odontológicas: o estado atual (Ceramic in dentistry: current situation) ,Rev. Araçatuba, SP

18. Moura, R. B. B.; Santos, T. C. R. Sistemas cerâmicos metal free: tecnologia CAD/CAM - revisão de literatura. Interd, n.8,v.1,p.220-226,2015.

19. Ogawa T, Ogimoto T, Koyano K. Pattern of occlusal contacts in lateral positions: canine protection and group function validity inclassifying guidance patterns. J Prosthet Dent. N.1,v.80,p.67-74,1998.

20. Resinas compostas: estágio atual e perspectivas. João Maurício FERRAZ DA SILVA. Daniel MARANHA DA ROCHA. Estevão Tomomitsu KIMPARA. Eduardo Shigueyuki UEMURA. Revista Odonto n. 32, v.16,p.98-104, 2008.

21. YAP, A.U.J.; SAU C.W.; LYE K.W. Effects of finishing \polishing time on surface characteristics of toothcoloured restoratives. J Oral Rehabil. n.25, p.456-61, 1998. 